\title{
Labyrinthe
}

$40 \mid 2013$

Comme les abeilles

\section{Google, apiculteur cognitif}

Ariel Kyrou

\section{(apenEdition}

Journals

Édition électronique

URL : http://journals.openedition.org/labyrinthe/4330

DOI : $10.4000 /$ labyrinthe.4330

ISSN : 1950-6031

Éditeur

Hermann

Édition imprimée

Date de publication : 1 mars 2013

Pagination : 129-134

ISBN : 9782705688400

Référence électronique

Ariel Kyrou, «Google, apiculteur cognitif », Labyrinthe [En ligne], 40 | 2013, mis en ligne le 01 mars

2015, consulté le 21 avril 2019. URL : http://journals.openedition.org/labyrinthe/4330 ; DOI : 10.4000/ labyrinthe.4330

Propriété intellectuelle 


\section{Google, apiculteur cognitif}

Ariel KYroU

Qu'est-ce qui permet de faire un pont entre le monde de Google et le monde de l'apiculture et des abeilles ? Exposons un premier point, qui peut paraître une vérité d'évidence: la ruche est une création artificielle, une infrastructure. C'est un réceptacle qui va évoluer et se transformer, mais qui est conçu par des hommes puis géré par des hommes, même si le miel n'est pas fabriqué par ces mêmes hommes. À la base, il y a donc la création d'une infrastructure pour les abeilles. Il en est de même Google, qui naît autour de 1998, à l'université Stanford; chez Google, ce qui joue un rôle majeur, ce sont les serveurs, qui permettent de numériser l'intégralité de ce qui passe dans le réseau. À la base même de Google, on trouve donc la création d'une double infrastructure: les gigantesques fermes de serveur, faisant office de ruche peut-être, mais aussi bien-sûr le logiciel du moteur de recherche, pour permettre aux internautes de « butiner» des connaissances. On a donc un premier point commun: une infrastructure construite pour d'autres.

Réfléchissons pour continuer à la manière dont fonctionne Apple et à celle dont fonctionne Google. Les gens qui travaillent pour Apple sont persuadés d'avoir l'entière maîtrise de tout ce qu'ils font et proposent; ce sont des créateurs. Si Apple était un dieu, ce serait un dieu créateur, monothéiste, un Dieu qui créerait et assumerait qu'il crée des systèmes ou objets qui sont le must du must. Les responsables d'Apple se comportent d'ailleurs dans le travail comme des Empereurs, qui détiennent un certain type de vérité, qu'ils proposent sous forme de produits à des gens qui vont en faire leur beurre. Il n'y a pas de rapport de cause à effet: une fois que vous avez votre iPhone ou votre Macintosh, Apple disparaît. Les services clients d'Apple sont d'ailleurs assez faibles, voire je-m'en-foutistes. C'est un système fermé, créé par des gens formidables qui ont un sens du design et de l'ergonomie assez extraordinaires, mais qui sont dans la logique d'un créateur omnipotent, presque tyrannique car ne supportant pas la moindre contradiction. Apple est Dieu (sur son cloud), point à la ligne. 


\section{Labyrinthe, $n^{\circ} 40$}

La logique de Google est très différente. Larry Page et Sergueï Brin, les créateurs de Google, disent qu'ils sont simplement un relais. Ce qui est important, c'est que ce soient les internautes qui fassent, qui cherchent, qui utilisent. Plus il y aura d'internautes, via tous les types de supports, plus on sera content. Les gens de Google disent même qu'ils seront le relais de toute l'information de l'univers. C'est l'un de leurs grands claim: l'un est « Don't be evil », et le deuxième est: « Nous voulons être le relais de l'information du monde ». L'un des seuls processus lourds chez Google, c'est le recrutement: il y a une quinzaine voire une vingtaine d'étapes, et la dernière, c'est « to be polite », être bien poli, être éduqué, pour « laisser les gens faire», permettre que les gens puissent agir et se construire eux-mêmes, tout seuls, grâce aux services de Google. Google a pour ambition d'être partout présent dès que quelqu'un cherche de l'information, l'air de rien. C'est en ce sens-là que le dieu de Google est spinozien : ce dieu est partout, il est distribué. Ce qui va avec, c'est que les acteurs de Google comme ceux du monde internet prennent internet et le monde numérique comme une seconde nature, et non comme une machine, comme un dispositif fabriqué. Google crée une infrastructure permettant aux abeilles-internautes de chercher dans l'immensité de la toile, mais internet est une seconde nature. Certains intellectuels voient en internet un bébé, un nouveau-né, qui va grandir et devenir un quasi être vivant. Cela peut justifier l'utilisation de métaphores, là encore avec toutes les simplifications abusives quelles pourraient porter mais aussi leur puissance d'évocation ou d'éclaircissement. Ces gens-là et les intellectuels qui sont leur référence ont la conviction que la vie n'est pas réductible au carbone, ni à quelque matière que ce soit. C'est un credo fort: la vie est information, elle est organisation de la matière plus que matière. Les scientifiques spécialistes de la vie artificielle refusent que seul le carbone puisse créer la vie ; pour eux, ce qui importe est l'organisation de la matière. Il est des personnages qui pensent qu'un programme peut être vivant. Ça va très loin. La vie est information. Voilà tout un schéma, toute une architecture, qui peuvent nous inciter à tisser la métaphore.

On va faire un pas en plus sur cette voie, pour essayer de mieux la comprendre. Cette métaphore de l'apiculteur se justifie d'autant plus que Google a intérêt à ce qu'il y ait un maximum d'internautes qui utilisent son moteur, et cette utilisation doit se faire en toute liberté, sans penser à l'algorithme de Google, à ce qui va nous permettre de trouver des résultats à nos requêtes. Google va avoir de plus en plus d' « AdWords » 


\section{Google, apiculteur cognitif}

- c'est-à-dire de mots achetés aux enchères et séparés sur le moteur de la recherche de la recherche elle-même - qu'il va y avoir de gens qui vont utiliser ce moteur de recherche. Au contraire de Facebook, vous pouvez dire ce que vous voulez de Google, mener les recherches les plus cochonnes ou injurieuses, il n'interviendra jamais. Dans l'imaginaire du moteur Google, plus les internautes vont vivre leur vie, butiner partout sur la toile et ailleurs leurs connaissances, voire numériser, mettre des sons et des images, les échanger, plus ça va polliniser, et mieux ce sera pour lui et aussi pour la société. Une connaissance est un bien «non rival », comme un fichier, une conférence: quand je le donne, je ne le perds pas. Même si je ne crée pas de la richesse immédiate, financière, une connaissance que je donne - c'est le principe même de l'éducation - enrichit la société. Pour les gens de Google, le travail qu'ils font, en essayant d'être de simples relais, de ne rien imposer, en laissant les abeilles butiner, c'est quelque chose qui est éminemment démocratique, mais aussi quelque peu méritocratique. De même que l'intérêt de l'apiculteur est que ça pollinise, au maximum, partout, de même l'écosystème naturel du web va d'autant plus servir à la fois les abeilles-internautes et Google que l'on va partager, s'échanger, sans aucune contrainte de propriété intellectuelle; c'est là l'intérêt et l'ambiguïté de Google, qui fait de l'argent grâce aux recherches des internautes - comme l'apiculture, avec le miel, au fond: est-ce honteux ou pas, c'est à discuter. En tout cas, Google récolte d'autant plus de miel que les abeilles butinent les connaissances. Cette logique est évidente. D’aucuns pensent même que Google participe ainsi du «bien commun ».

Le moteur de recherche Google et son algorithme PageRank fonctionnent de façon intéressante: quand on tape un mot, on trouve des occurrences du mot; la force de l'algorithme est qu'il prend en compte la popularité des sites, avant de les proposer à l'internaute. Il considère tout lien hypertexte vers un texte comme un « vote» pour lui. Plus tel site aura de votes, plus il sera valorisé. Plus il y a de liens hypertextes pointant vers un site, plus il va monter dans la « valeur» que va lui accorder le moteur de recherche. Les sites les plus réputés ont une valeur, en termes de votes, plus forte que ceux qui sont moins pointés que les autres. Ce moteur fonctionne donc en valorisant les liens, le partage; le fait que les gens sont acteurs et vont à la chasse aux sites les plus intéressants en termes de connaissance, voilà qui est valorisé par Google. Ceux qui 


\section{Labyrinthe, $n^{\circ} 40$}

sont les plus actifs sont les plus valorisés au sein même de l'écosystème de Google et, plus largement, d'internet.

En termes de tendance, Google a une spécificité, qui est de savoir faire travailler les autres, comme un apiculteur doit réussir à ce que ses abeilles soient bien, pollinisent le plus possible. Prenons l'exemple du système d'exploitation (operating system) Android, apparu en 2007 : a contrario d'Apple veut avoir la maîtrise absolue des tâches nobles, des fonctions essentielles de son iPhone, et laisser les basses-œuvres à des sous-traitants, Google, lui, a fait quelque chose de rigoureusement opposé à ce qui se faisait dans le monde du mobile. Il n'a pas créé de téléphone, mais fait un système ouvert, en open source, et a décidé de proposer à tous les acteurs d'utiliser ce système d'exploitation. Google se définit comme une ressource, telle la reine-mère, qui va vous permettre de faire ce que vous voulez. Le monde du mobile s'est dit au début que ce choix était fou, que ça ne marcherait jamais. Aujourd'hui, il se vend bien plus de smartphone sous système d'exploitation Android que de iPhone sous iOS. Il y a certes des échecs: Google a lancé un appareil sous sa marque propre, Nexus, qui est très loin d'avoir eu le succès des smartphones Samsung sous Android. Ce qui marche, c'est quand ils laissent faire, quand ils laissent faire à leur place. L'histoire de l'Open Handset Alliance, qui a réuni les fabricants auxquels Google a « offert » son OS Android, est intéressante: c'est une démarche qui n'est pas du même ordre que le capitalisme de grand-papa, type General Motors. On est là dans quelque chose qui tranche dans la manière de faire et de construire ; on accepte désormais d'être dessaisi par d'autres, on accepte ce jeu, on accepte la multiplicité de ce qui va naître à partir des sources dont j'ai été le pourvoyeur.

Lorsqu'on parle de société insectoïde, on parle aussi beaucoup de traces: des traces qui sont laissées et qui permettent d'aller sur telle ou telle piste, fructueuse en pollen ou autres. Lorsqu'on s'intéresse à ce monde numérique, quelque chose qui ressort assez couramment est le fait que, effectivement, Google fait une part de son miel en gardant la trace de tout ce que font les internautes utilisant ses services. On peut effacer ses traces; mais le moteur retient ce que vous avez cherché et vous propose des termes à partir des premiers mots, et puis aussi de nouveaux services ou des publicités censés correspondre à vos goûts et besoins. Dans un univers saturé d'ondes, de géolocalisation, avec le GPS en voiture, etc., il y a certes une logique de partage entre individus, 


\section{Google, apiculteur cognitif}

mais aussi une logique de traces et de suivi des personnes « à la trace ». Google vous connaît mieux que vous-même. Et ce n'est sans doute pas une bonne nouvelle pour notre libre-arbitre ! Pour le coup, sur ces traceslà, il est facile et sans doute nécessaire de tirer la métaphore de notre rapport aux insectes vers le négatif. 
"Cher enfant, lui dit-elle, essuie enfin tes larmes;

Tu connais ton destin. Eurydice autrefois

Accompagnait les chœurs des nymphes de ces bois;

Elles vengent sa mort : toi, fléchis leur colère :

On désarme aisément leur rigueur passagère.

Sur le riant Lycée, où paissent tes troupeaux,

Va choisir à l'instant quatre jeunes taureaux;

Choisis un nombre égal de génisses superbes,

Qui des prés émaillés foulent en paix les herbes;

Pour les sacrifier élève quatre autels;

Et, les faisant tomber sous les couteaux mortels,

Laisse leurs corps sanglants dans la forêt profonde.

Quand la neuvième aurore éclairera le monde,

Au déplorable époux dont tu causas les maux,

Offre une brebis noire et la fleur des pavots;

Enfin, pour satisfaire aux mânes d'Eurydice,

De retour dans les bois, immole une génisse. "

Elle dit : le berger dans ses nombreux troupeaux

Va choisir à l'instant quatre jeunes taureaux;

Immole un nombre égal de génisses superbes,

Qui des prés émaillés foulaient en paix les herbes.

Pour la neuvième fois quand l'aurore parut,

Au malheureux Orphée il offrit son tribut,

Et rentra plein d'espoir dans la forêt profonde.

Ô prodige! Le sang, par sa chaleur féconde,

Dans le flanc des taureaux forme un nombreux essaim;

Des peuples bourdonnants s'échappent de leur sein,

Comme un nuage épais dans les airs se répandent,

Et sur l'arbre voisin en grappes se suspendent.

Virgile, Géorgiques, op. cit., v. 531-558. 